Gynäkologe 2021 · 54:381

https://doi.org/10.1007/s00129-021-04805-5

Angenommen: 12. April 2021

(c) Springer Medizin Verlag $\mathrm{GmbH}$, ein Teil von Springer Nature 2021

Als dieses Heft konzipiert wurde, waren die hohe Aktualität und Brisanz des Themas noch nicht absehbar. Die im Laufe des Jahres 2020 sich entwickelnde, uns auf allen Ebenen unseres Lebens fesselnde SARS-CoV2-Pandemie (severe acute respiratory syndrome coronavirus type 2) lag damals noch in der unbekannten Zukunft.

Aber Fragen der Hygiene forderten bereits vorher unsere Aufmerksamkeit. Unsere Verantwortung für den Schutz der Patientinnen und unserer Mitarbeiter vor Infektionen innerhalb des Krankenhauses verlangte bereits vor der Pandemie die Schaffung geeigneter Strukturen innerhalb unserer Frauenkliniken. Dabei war unbestritten, dass es immer wieder hygienische Probleme mit nosokomialen Infektionen, auch unter Beteiligung von multiresistenten Keimen, gegeben hat und auch noch gibt - trotz des im Jahr 2001 in Kraft getretenen Infektionsschutzgesetzes, mit dem der Gesetzgeber das Problem in breit angelegter Form angehen wollte. Gleiches gilt für die erstmals im Jahr 2008 von der Bundesregierung formulierte „Deutsche Antibiotika-Resistenzstrategie“. Das Auftreten von nicht mehr behandelbaren Mikroorganismen soll und muss verhindert werden.

\section{》) Das Auftreten von nicht mehr behandelbaren Mikroorganismen muss verhindert werden}

Empfehlungen der Kommission für Krankenhaushygiene und Infektionsprävention am Robert-Koch Institut galt es umzusetzen. Hygienepläne sind

\author{
Matthias Sauter ${ }^{1}$ Ricardo E. Felberbaum ${ }^{2}$ \\ ${ }^{1}$ Abteilung für Hygiene und Infektiologie, Klinikverbund Allgäu gGmbH, Kempten, Deutschland \\ ${ }^{2}$ Klinikum Kempten, Klinik für Gynäkologie und Geburtshilfe, Klinikverbund Allgäu gGmbH, Kempten, \\ Deutschland
}

\title{
Hygiene in der Frauenheilkunde
}

erarbeitet worden und mehr Hygienefachpersonal ist eingestellt worden. Aber hat das gereicht? Und sind die Vorgaben auf die Bedürfnisse der Kliniken für Frauenheilkunde und Geburtshilfe angepasst worden? Epidemiologische Methoden, wie das Führen von Infektionsstatistiken und Surveillance, ebenso regelmäßige Begehungen der einzelnen Stationen und Funktionsbereiche, sollen eine regelmäßige Analyse der hygienischen Zustände erlauben. Und wo stehen wir da in unseren Frauenkliniken? Eine Bestandsaufnahme schien angezeigt.

Und dann kam COVID-19. Nie trugen unsere Fachabteilungen für Hygiene eine höhere Verantwortung, waren stärker gefragt und befragt als während dieser weltumspannenden Lage. Daher erscheint es verständlich, dass dieses Heft nun die grundlegenden Hygienestandards in der operativen Gynäkologie, der gynäkologischen Praxis, der Geburtshilfe und der Neonatologie beschreibt und diskutiert, aber eben auch immer unter dem Aspekt - bzw. vor dem Hintergrund - der aktuellen Pandemie. Daneben werden auch die organisatorischen Probleme, die sich in den Vorbereitungen auf die erste und zweite Welle der Pandemie für alle Krankenhäuser ergeben haben, dargestellt. Die Geburtshilfe nahm dabei, neben der Notfallmedizin, insofern einen Sonderplatz ein, als dass hier eine Reduktion elektiver diagnostischer und therapeutischer Maßnahmen nicht möglich war. Gleiches gilt für die neonatologischen Intensivstationen. Diese kritischen Versorgungsstrukturen mussten ungebremst durch die Pandemie gelenkt werden.

Vieles von dem, was wir in der Herausforderung und Überforderung durch die Pandemie erleben und erlernen konn- ten, sollten wir auch nach Überwindung der Pandemie in den klinischen Alltag hinübernehmen.

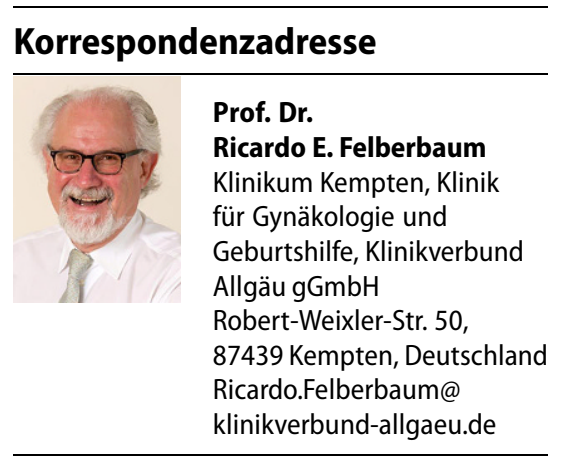

Interessenkonflikt. M. Sauter und R.E. Felberbaum geben an, dass kein Interessenkonflikt besteht. 